\title{
Comparison of Primary Open Angle Glaucoma Patients in Rural and Urban Ghana
}

Andrew W. Francis ${ }^{1}$, Michael E. Gyasi², Martin Adjuik², Emmanuel Kesse², Yifan Chen³, Rhys S.R. Harrison ${ }^{4}$, R.A. Kodjo ${ }^{2}$

1. Department of Ophthalmology and Visual Sciences, University of Illinois College of Medicine at Chicago, Chicago, IL, USA

2. Emmanuel Eye Centre, Accra, Ghana

3. Yale University, New Haven, CT, USA

4. Department of Ophthalmology, Royal United Hospital Bath NHS Trust, UK.

\begin{abstract}
:
Purpose: To compare the clinical features of glaucoma patients who present at a rural hospital in North Eastern Ghana and an urban hospital in the capital city of Accra.

Methods: This is a multi-center retrospective case series involving records of newly diagnosed glaucoma patients with emphasis on primary open angle glaucoma (POAG). Information collected included basic demographic data, intraocular pressures and optic disc measurements.

Results: A total of 949 patients (437 rural; 512 urban; 1868 eyes) were included. Rural vs. urban comparisons, respectively: mean age, $53.2 \pm 16.3$ vs. $54.5 \pm 16.4$ years; male: female ratio, $3: 2$ vs. $1: 1 ;$ POAG, $78.1 \%$ vs. $50.6 \%$; POAG suspect, $10.3 \%$ vs. $41.9 \%$; IOP, $39.2 \pm 7.1$ vs. $31.8 \pm 7.3 \mathrm{mmHg}$; bilateral blindness, $34.1 \%$ vs. $17.5 \%$; uniocular blindness, $52.2 \%$ vs. $32.9 \%$. Females at the rural hospital were twice as likely to present blind in at least one eye (OR 2.04, CI 1.36 - 3.07, p<0.001).

Conclusions: Patients with POAG at the rural hospital present with more advanced disease characteristics.

Keywords: glaucoma, open angle,Ghana, Urban, rural

DOI:http://dx.doi.org/10.4314/ahs.v14i3.32
\end{abstract}

\section{Introduction}

Primary open angle glaucoma (POAG) is the most common cause of irreversible adult blindness in African nations and imposes a significant burden both on individuals and society. ${ }^{1,2}$ POAG is a chronic optic neuropathy with characteristic patterns of optic nerve injury and visual field loss.$^{1-3}$ Elevated intraocular pressure (IOP) is the most important risk factor for POAG progression and is the primary therapeutic target.

In prevalence studies, the diagnosis of POAG best conforms to the definition from the Working Group for Defining Glaucomas in Prevalence Studies, also referred to as the International Society of Geographical and Epidemiological Ophthalmology (ISGEO), ${ }^{4}$ which characterizes POAG by structural and functional methods. Diagnosis of POAG is determined by structural changes in the optic nerve and damage to the visual fields which are determined by both the level

\section{Corresponding Author: \\ Michael E. Gyasi \\ Emmanuel Eye Centre \\ P.O. Box GP8967, Accra, Ghana \\ Tel: +233-246-866580 \\ Email: mikegyasi@yahoo.co.uk.}

of IOP and the optic nerve axon susceptibility to injury. The presence of normal tension glaucoma (NTG), with optic disk cupping and visual field defects despite IOP measurements in the statistically normal range, is evidence that predisposition to optic nerve injury is at least partially independent of elevated IOP.

Recent population studies in Ghana estimate $7.7 \%$ of the population over the age of 30 years and $8.5 \%$ over the age of 40 years suffer from this disease. ${ }^{5}$ This estimate is comparable to population studies in other African-descended populations such as St. Lucia, Barbados, Ethiopia and South Africa. ${ }^{6-9}$ In a previous study of patients in the North Eastern region of Ghana, records of 891 eyes of 446 patients were reviewed revealing $98.4 \%$ of patients suffered from POAG with 8.3\% classified as NTG patients. More than one third of all patients presented bilaterally blind and nearly half were blind in at least one eye. ${ }^{10}$ Lowering the IOP remains the only proven therapy for stabilization of the characteristic optic nerve excavation and visual field changes that define the severity of this disease. ${ }^{10-12}$

A thorough review of the published literature demonstrated no study that has compared the presentation of POAG in rural and urban settings in Ghana. We therefore sought to compare the clinical features of glaucoma patients who present at a rural hospital in North Eastern Ghana and an urban hospital in the capital city of Accra to determine if significant 
differences in clinical presentation are present which may alter screening guidelines and treatment of patients with POAG.

\section{Materials and methods \\ Study Design}

This is a multi-center retrospective case series involving the review of clinical records at two separate hospitals. All newly diagnosed POAG or POAG suspects were included. Patients with secondary glaucomas were also recorded. The first center is a large public eye clinic (Bawku hospital) located in the mainly rural Upper East Region of Ghana while the second center is another large public eye clinic (the Emmanuel Eye Centre) located in Ghana's national capital, Accra. Information collected included basic demographic data, measured IOP at presentation and optic disc measurements.

\section{Outcome measures}

The principal outcome measure in this study was the diagnosis and clinical features of POAG, which was determined in all cases by experienced ophthalmologists using standard ophthalmologic equipment. All cases had their IOP measured with the slit lamp-mounted Goldman Applanation Tonometer and stereoscopic optic disc assessment using either the 78D or 90D Volk indirect fundus lenses.

\section{Definitions and Diagnostic Criteria}

In this study, the definition of POAG conformed to the recommendations of the Working Group for Defining Glaucoma in Prevalence Studies (International Society of Geographical and Epidemiological Ophthalmology [ISGEO]) (table 1). ${ }^{4}$ A POAG patient herein was defined as a person who met any of the
ISGEO categorizations in either one or both eyes. For this study, the diagnosis of POAG was made primarily by characteristic structural defects in the optic nerve head rather than functional deficits in the visual fields as this allowed for standardized comparisons of optic nerve head changes over time. Types of secondary glaucomas and gonioscopic measurements were recorded, but the emphasis of this study was POAG. For the purpose of this study, a NTG patient was defined as POAG in which IOP was measure $\leq 21 \mathrm{mmHg}$ in the absence of anti-hypertensive medications.

\section{Exclusion criteria}

Patients with a history of cataract surgery, glaucoma filtering procedures, congenital glaucoma or those on glaucoma medications were excluded. Patients with a secondary glaucoma were recorded, but further statistical studies were not performed.

\section{Statistical Analysis}

All the required information was entered onto a pre-designed Microsoft excel template and analysis done using the Epi Info Software (Revision 2, January 302003 Centre for Disease Control and Prevention, Atlanta Georgia). Descriptive statistics were used to summarize the characteristics of the studied subjects. Ages of the patients were summarized using the mean, median and standard deviation. Comparison of strength of associations was done using $\mathrm{Chi}^{2}$ test of significance. All statistical tests were two-sided with an alpha level of $<0.05$ considered statistically significant.

\section{Results}

A total of 949 patients (437 rural; 512 urban; 1868 eyes) were included (table 1).

Table 1: ISGEO Categorizations used in Glaucoma prevalence studies (modified)

\begin{tabular}{|l|l|}
\hline Category & Structural Abnormality \\
\hline 1 & $\begin{array}{l}\text { Eyes with a CDR or CDR asymmetry } \geq 97.5^{\text {th }} \text { percentile for the normal population, or } \\
\text { NRR width reduced to } \leq 0.1 C D R \text { (between } 11 \text { to } 1 \text { o'clock or } 5 \text { to } 7 \text { o'clock). }\end{array}$ \\
\hline 2 & $\begin{array}{l}\text { Advanced structural damage with CDR or CDR asymmetry } \geq 99.5^{\text {th }} \text { percentile for the } \\
\text { normal population }\end{array}$ \\
\hline 3 & $\begin{array}{l}\text { Optic disc not seen but visual acuity }<3 / 60 \text { and IOP }>99.5^{\text {th }} \text { percentile or visual acuity } \\
<3 / 60 \text { with evidence of glaucoma filtering surgery or medical records of glaucomatous } \\
\text { visual morbidity }\end{array}$ \\
\hline
\end{tabular}


Of the 437 patients in the rural hospital, 78.1\% were diagnosed with POAG in at least one eye, 10.3\% were diagnosed as POAG suspects and 10.3\% were diagnosed with NTG. At the urban hospital 50.6\% of patients were diagnosed with POAG, $41.9 \%$ were identified as POAG suspects and 3.9\% were diagnosed with NTG. Right and left eyes were equally affected in both urban and rural cases. Two out of every three patients diagnosed at the rural hospital were men; in contrast, there was nearly equal division of males and females diagnosed at the urban center (table 2).

Table 2: Glaucoma Types

\begin{tabular}{|c|c|c|c|c|}
\hline \multirow[t]{2}{*}{ Diagnosis } & \multicolumn{2}{|c|}{ Rural } & \multicolumn{2}{|c|}{ Urban } \\
\hline & $\%$ & $\mathrm{No}$ & $\%$ & No \\
\hline POAG & 78.1 & 668 & 50.6 & 513 \\
\hline POAGS & 10.3 & 88 & 41.9 & 424 \\
\hline NTG & 10.3 & 88 & 3.9 & 40 \\
\hline PAC & $==$ & $==$ & 1.2 & 12 \\
\hline Secondary Glaucomas & 1.3 & 11 & 2.4 & 24 \\
\hline TOTAL & 100 & 855 & 100 & 1013 \\
\hline
\end{tabular}

Table 2: POAG - primary open angle glaucoma, POAGS - primary open angle glaucoma suspect, NTG - normal tension glaucoma, PAC - primary angle closure

The mean ages between the two groups were statistically similar (figure 1). In the rural setting the mean age of initial evaluation was $53.2 \pm 16.3$ years (median 56; range 9-87 years) vs. 54.5 years \pm 16.4 years (median 57; range 10-96 years) at the urban hospital.
In total, $23.6 \%$ of patients in the rural setting were diagnosed under the age of 40 years versus $20.2 \%$ at the urban hospital. The absolute number of patients diagnosed under the age of 40 years was identical in both settings (103 patients).

Figure 1: Age distribution among Urban and Rural glaucoma patients.

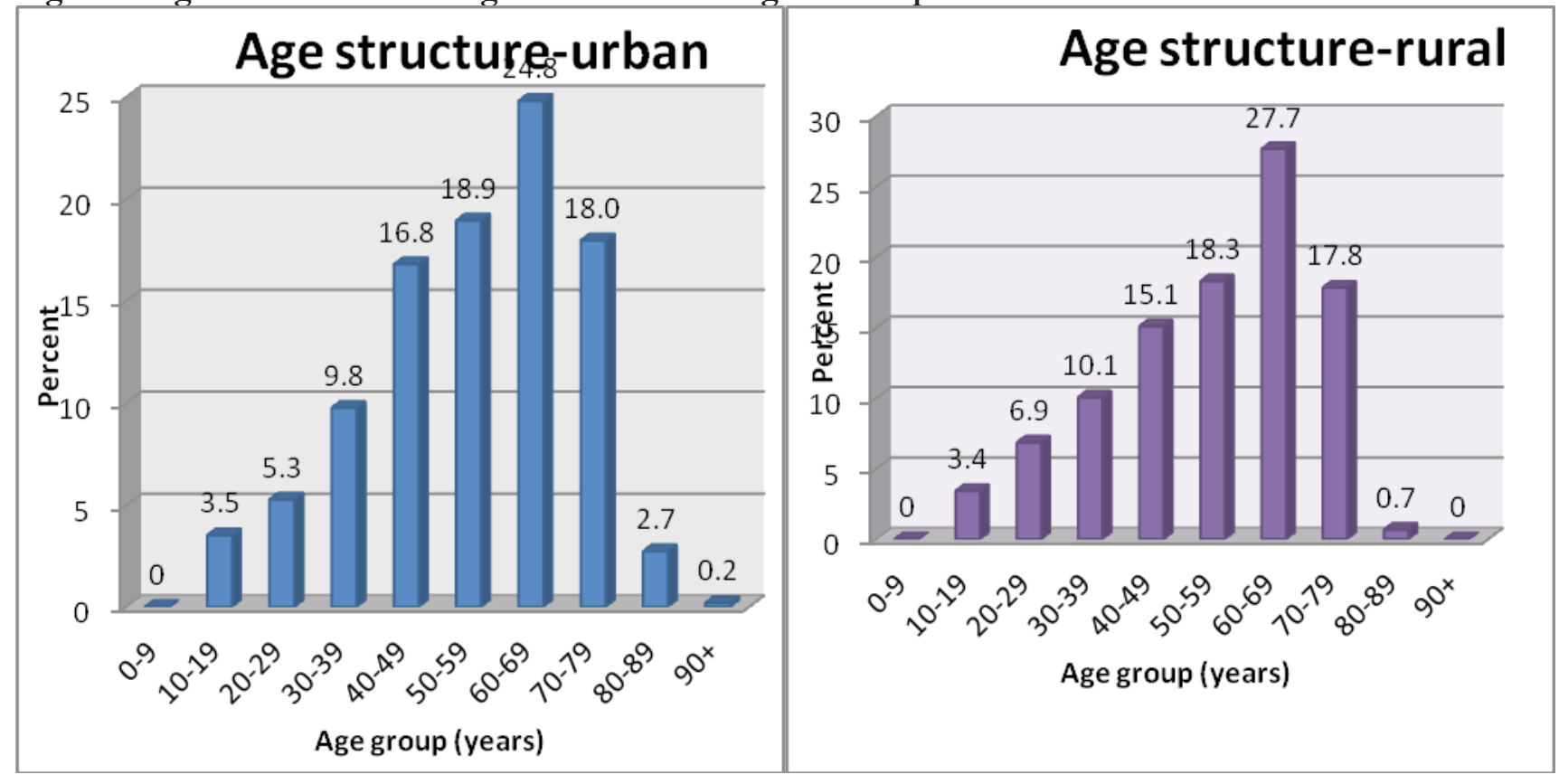


The mean IOP of POAG patients was $39.2 \pm$ $7.1 \mathrm{mmHg}$ in the rural hospital (range 22-69 $\mathrm{mmHg}$ ) vs. $31.8 \pm 7.3 \mathrm{mmHg}$ (range $22-72 \mathrm{mmHg}$ ) in the urban hospital $(\mathrm{p}<0.01)$. Of all POAG patients diagnosed in the rural hospital, $62 \%$ presented for treatment with an IOP $>40 \mathrm{mmHg}$ versus only $6.8 \%$ of patients in the urban hospital $(\mathrm{p}<0.001)$ (table 3).

Table 3: Basic Demographics and Clinical Exam Findings

\begin{tabular}{|c|c|c|c|c|c|}
\hline & \multicolumn{2}{|l|}{ Rural } & \multicolumn{2}{|l|}{ Urban } & \multirow[t]{2}{*}{ P Value } \\
\hline & $\%$ & No & $\%$ & No & \\
\hline Patients & - & 437 & - & 512 & \\
\hline Eyes & - & 855 & - & 1013 & \\
\hline Male/Female & $65.5 / 34.5$ & $286 / 151$ & $50.7 / 49.3$ & $260 / 252$ & $<0.01$ \\
\hline OD/OS & $50.1 / 49.9$ & $428 / 427$ & $49.9 / 50.1$ & $505 / 508$ & NS \\
\hline Mean Age (yrs) & - & 53.2 & - & 54.5 & NS \\
\hline $\mathrm{SD}$ & - & 16.3 & - & 16.4 & \\
\hline Median & - & 56 & - & 57 & \\
\hline Range & - & $9-87$ & - & $10-96$ & \\
\hline Age $\leq 40 \mathrm{yrs}$ & 23.6 & 103 & 20.2 & 103 & NS \\
\hline TOP (all) $\mathrm{mmHg}$ & - & $35.5 \pm 10.4$ & - & $29.4 \pm 8.5$ & $<0.01$ \\
\hline IOP (POAGS) & - & $39.2 \pm 7.1$ & - & $31.8 \pm 7.3$ & $<0.01$ \\
\hline Range & - & $22-69$ & - & $22-72$ & \\
\hline $\mathrm{IOP} \geq 40 \mathrm{mmHg}$ & 62.2 & & 6.8 & & $<0.001$ \\
\hline Uniocular Blindness & 52.2 & 228 & 32.9 & 168 & $<0.01$ \\
\hline Bilateral blindess & 34.1 & 149 & 17.5 & 90 & $<0.01$ \\
\hline
\end{tabular}

Table 3: SD - standard deviation, POAGS - primary open angle glaucoma suspect, IOP - intraocular pressure, NS - not significant

At the rural hospital, $34.1 \%$ presented bilaterally blind (BCVA $<3 / 60$, Logmar < 1.3) and 52.2\% presented blind in at least one eye vs. $17.5 \%$ and $32.9 \%$ at the urban hospital, respectively (both $\mathrm{p}<0.01$ ). In the rural setting females were twice as likely to present blind in at least one eye (OR 2.04, CI $1.36-3.07$, p <0.001), but in the urban setting, there was equal risk of blindness among sexes (OR 1.00, CI $0.74-1.33, \mathrm{p}=0.978$ ). 


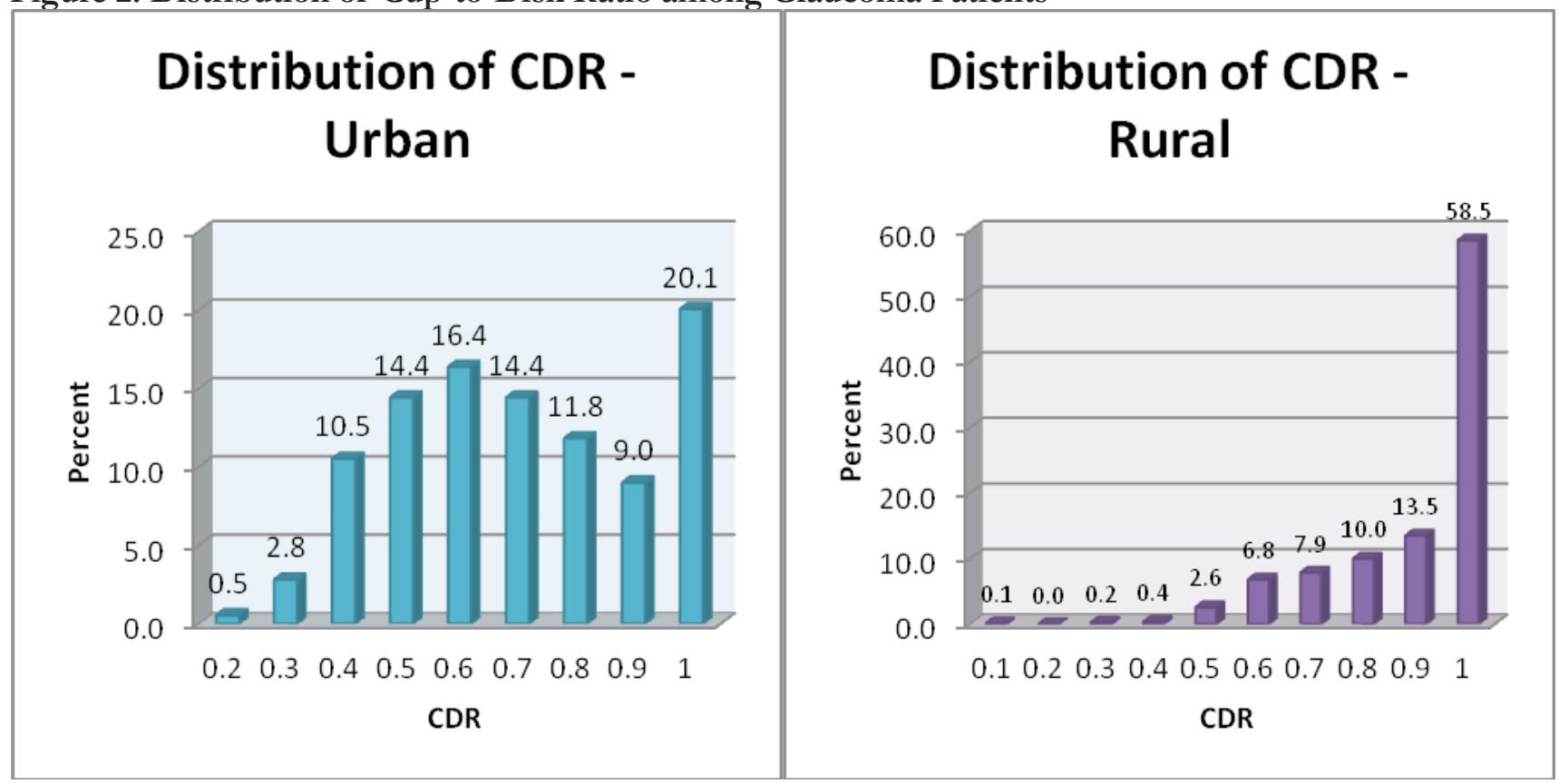

\section{Discussion}

The prevalence of POAG in Ghana ranks among the most affected countries in the world with estimates of $7.7 \%$ of the population over the age of 30 years and $8.5 \%$ over 40 years of age afflicted. ${ }^{5}$ To the best of our knowledge, no study in Ghana has yet compared the clinical features of POAG at a rural hospital and an urban hospital in the same manuscript. Our findings highlight the characteristics that have come to define POAG in many African populations: earlier onset, often before the age of 40 years, more severe clinical findings and a more aggressive clinical course with higher rates of blindness. Among whites aged 40 years or older, the estimated worldwide prevalence of POAG is between 1.1 and $2.1 \% .{ }^{13}$ Based on population studies in Black populations, the prevalence is at least 3 to 4 times higher with at least 4 times the likelihood of blindness. ${ }^{14}$ Estimations show prevalence increases with age, with some estimations showing a risk of blindness 15 times greater for affected individuals between $46-$ 65 years of age. ${ }^{15}$

The mean age of diagnosis in this study was 53.2 in the rural versus 54.5 years in the urban setting, which closely approximates hospital-based studies in Nigeria (52.7 years), Cameroon (53.3 years) and Ethiopia (51.9 years)..$^{16-18} \mathrm{~A}$ higher percentage of patients were diagnosed with POAG in the rural hospital versus the urban hospital. At the rural hospital, $78.1 \%$ were diagnosed with POAG and $10.3 \%$ as POAG suspects versus $50.6 \%$ and $41.9 \%$ at the urban center, respectively. Later presentation to the health care system is the most likely explanation for this variance. Patients at the rural hospital presented with higher IOP (39.2 vs. $31.8 \mathrm{mmHg}, \mathrm{p}<0.01)$, more optic nerve excavation on slit-lamp examination (figure 2) and higher rates of uniocular and bilateral blindness (table 3).

The percentage of patients diagnosed with NTG in the rural setting was $10.3 \%$ versus only $3.9 \%$ in the urban setting. This finding should likely be taken in the context of the significant overlap between IOP distribution curves in glaucomatous and non-glaucomatous eyes. In general, an IOP screening value $>21 \mathrm{mmHg}$ has little clinical significance in the absence of confirmatory examination findings that support the diagnosis of POAG. Several studies have shown that between $30-50 \%$ of glaucoma patients with characteristic optic nerve neuropathy and/or visual field changes have initial screening IOPs $<22 \mathrm{mmHg}^{11,19-21}$ IOP is also known to vary widely throughout the day, with fluctuations of $10 \mathrm{mmHg}$ or more in glaucoma patients, but only $2-6 \mathrm{mmHg}$ in normal subjects. ${ }^{22}$ The higher percentage NTG patients identified at the rural hospital may manifest statistically higher pressures at different examination times.

The nearly 3:2 male to female ratio of POAG diagnose in the rural setting appears to be secondary to presentation bias rather than intrinsic susceptibility to disease. Studies in Black populations and elsewhere are conflicted in regards to the incidence of glaucoma according to gender. In the Barbados eye study, ${ }^{7}$ males had a higher rate of POAG, in St Lucia ${ }^{6}$ women outnumbered males, but in the Baltimore Eye study ${ }^{14}$ 
no statistical associations were found at all. The likely explanation of the gender variance in this study, specifically in the rural setting, is an underreporting of females with disease. Hospital based studies previously mentioned in the North Eastern region of Ghana ${ }^{10}$, Nigeria ${ }^{16}$ and Ethiopia ${ }^{18}$ also reported nearly a 2:1 male ratio, likely for the same reason.

A trend of more severe structural damage was seen in the rural setting (figure 2). Nearly $60 \%$ of patients who presented in the rural setting had an estimated CDR of $>0.9$ compared to only $20 \%$ in the urban setting. This finding matches the IOP distribution patterns and blindness patterns in these two study groups. Given the mean presenting age is similar between these two populations, the cause of this disparity in optic nerve head damage cannot be accounted for by presenting age alone. This may represent selection bias, where patients with severe visual field defects are more likely to present at the rural hospital in greater numbers once visual changes become readily apparent. Urban patients may be more likely to seek routine screening with earlier identification of features consistent with POAG.

In summary, patients at the rural hospital in North Eastern Ghana appear to present at a more severe stage of POAG and are at risk of poorer outcomes than patients in the urban hospital in Accra, Ghana. Females may be at a higher risk based on our findings. Further studies are necessary, especially in Ghana's rural population.

\section{Limitations}

This study was a comparative case series of patients and only associations can be drawn between groups. Evidence was collected from two large outpatient referral centers and is not reflective of the entire Ghana population. We were not able to account for diurnal variations in IOP. Pachymetry measurements were not recorded and IOP averages may be under or overestimated. We elected not to analyze or compare visual field findings due to the wide differences in field assessment techniques used, instead focusing on characteristic structural changes in the optic nerve to diagnose POAG. The availability of OCT or fundus photography may have reduced bias in determining optic nerve head defects.

\section{Financial Support:}

None

\section{Conflict of Interest:}

No conflicts of interest are associated with this study.

\section{Statement about Conformity with Author Information:}

This study is in accordance with Health Insurance Portability and Accountability Act regulations and followed the tenets of the Declaration of Helsinki and received an approval from institutional review board (IRB) at the Emmanuel Eye Centre, Accra, Ghana. As corresponding author, I take full responsibility for the integrity of this work and the decision to publish.

\section{References}

1.Racette L, Wilson MR, Zangwill LM, Weinreb RN, Sample PA. Primary open-angle glaucoma in blacks: a review. Surv Opbthalmol 2003;48:295-313.

2.Leske MC. The epidemiology of open-angle glaucoma: a review. Am J Epidemiol 1983;118:166-191.

3.Quigley HA, Broman AT. The number of people with glaucoma worldwide in 2010 and 2020. Br J Ophthalmol 2006;90:262-267.

4.Foster PJ, Buhrmann R, Quigley HA, Johnson GJ. The definition and classification of glaucoma in prevalence surveys. Br J Ophthalmol 2002;86:238-242.

5.Ntim-Amponsah CT, Amoaku WM, Ofosu-Amaah S, et al. Prevalence of glaucoma in an African population. Eye (Lond) 2004;18:491-497.

6.Mason RP, Kosoko O, Wilson MR, et al. National survey of the prevalence and risk factors of glaucoma in St. Lucia, West Indies. Part I. Prevalence findings. Ophthalmology 1989;96:1363-1368.

7.Leske MC, Connell AM, Schachat AP, Hyman L. The Barbados Eye Study. Prevalence of open angle glaucoma. Arch Ophthalmol 1994;112:821-829.

8.Buhrmann RR, Quigley HA, Barron Y, West SK, Oliva MS, Mmbaga BB. Prevalence of glaucoma in a rural East African population. Invest Ophthalmol Vis $S_{c i}$ 2000;41:40-48.

9.Rotchford AP, Johnson GJ. Glaucoma in Zulus: a population-based cross-sectional survey in a rural district in South Africa. Arch Opbthalmol 2002;120:471478.

10.Gyasi M, Amoako W, Adjuik M. Presentation patterns of primary open angle glaucomas in north eastern ghana. Ghana Med J 2010;44:25-30.

11.Leske MC, Heijl A, Hussein M, et al. Factors for glaucoma progression and the effect of treatment: the early manifest glaucoma trial. Arch Ophthalmol 2003;121:48-56.

12.Comparison of glaucomatous progression between untreated patients with normal-tension glaucoma and patients with therapeutically reduced intraocular 
pressures. Collaborative Normal-Tension Glaucoma Study Group. Am J Ophthalmol 1998;126:487-497.

13.Friedman DS, Wolfs RC, O'Colmain BJ, et al. Prevalence of open-angle glaucoma among adults in the United States. Arch Ophthalmol 2004;122:532-538.

14.Tielsch JM, Sommer A, Katz J, Royall RM, Quigley HA, Javitt J. Racial variations in the prevalence of primary open-angle glaucoma. The Baltimore Eye Survey. JAMA 1991;266:369-374.

15.Javitt JC, McBean AM, Nicholson GA, Babish JD, Warren JL, Krakauer H. Undertreatment of glaucoma among black Americans. N Engl J Med 1991;325:14181422.

16.Omoti AE, Osahon AI, Waziri-Erameh MJ. Pattern of presentation of primary open-angle glaucoma in Benin City, Nigeria. Trop Doct 2006;36:97-100.

17.Eballé AO, Mvogo CE, Koki G, et al. Prevalence and causes of blindness at a tertiary hospital in Douala,
Cameroon. Clin Ophthalmol 2011;5:1325-1331.

18. Melka F, Alemu B. The pattern of glaucoma in Menelik II Hospital Addis Ababa, Ethiopia. Etbiop Med J 2006;44:159-165.

19.Bergeå B, Bodin L, Svedbergh B. Impact of intraocular pressure regulation on visual fields in openangle glaucoma. Ophthalmology 1999;106:997-1004; discussion 1004-1005.

20.Liu JH, Kripke DF, Twa MD, et al. Twenty-four-hour pattern of intraocular pressure in the aging population. Invest Ophthalmol V is Sci 1999;40:2912-2917.

21.Realini T, Barber L, Burton D. Frequency of asymmetric intraocular pressure fluctuations among patients with and without glaucoma. Ophthalmology 2002;109:1367-1371.

22.Asrani S, Zeimer R, Wilensky J, Gieser D, Vitale S, Lindenmuth $\mathrm{K}$. Large diurnal fluctuations in intraocular pressure are an independent risk factor in patients with glaucoma. J Glaucoma 2000;9:134-142. 